\title{
ON VECTOR MEASURES
}

\author{
BY \\ J. DIESTEL AND B. FAIRES
}

\begin{abstract}
The four sections of this paper treat four different but somewhat related topics in the theory of vector measures. In $\$ 1$ necessary and sufficient conditions for a Banach space $X$ to have the property that bounded additive $X$-valued maps on $\sigma$-algebras be strongly bounded are presented, namely, $X$ can contain no copy of $l_{\infty}$. The next two sections treat the Jordan decomposition for measures with values in $L_{1}$-spaces on $c_{0}(\Gamma)$ spaces and criteria for integrability of scalar functions with respect to vector measures. In particular, a different proof is presented for a result of $D$. R. Lewis to the effect that scalar integrability implies integrability is equivalent to noncontainment of $c_{0}$. The final section concerns the Radon-Nikodym theorem for vector measures. A generalization of a result due to E. Leonard and K. Sundaresan is given, namely, if a Banach space $X$ has an equivalent very smooth norm (in particular, a Fréchet differentiable norm) then itsdual has the Radon-Nikodym property. Consequently, a $C(\Omega)$ space which is a Grothendieck space (weak-star and weak-sequential convergence in dual coincide) cannot be renormed smoothly. Several open questions are mentioned throughout the paper.
\end{abstract}

The present paper contains results on various aspects of the general theory of vector-valued measures. It proceeds in four sections which are unrelated to each other except for their general relationship to the topic of the title. A brief outline of the results of each section is presented below-a more complete discussion of the sections is delayed (largely because of their disconnected nature) until the sections themselves.

$\$ 1$ is concerned with the theory of strongly bounded vector measures. The main result of this section (Theorem 1.1) provides criteria for a Banach space $X$ to possess the property that every $X$-valued bounded additive map with values in $X$ be strongly bounded. This theorem sharpens the classical Pettis theorem on weakly countably additive set functions and allows a sharpening of several other related results.

$\$ 2$ is concerned with the Jordan decomposition of vector measures with values in a Banach lattice. The results of this section are necessarily meager: not much is possible. Our most precise results are in case the range space is an abstract $L$ space or $c_{0}$. A few remarks are also made concerning the range of certain vector measures.

$\$ 3$ deals with the integrability of certain scalar functions with respect to a vector measure. Utilizing the series representation of a scalar function and its integral, a result of D. R. Lewis is generalized. Also, a criterion for integrability

Received by the editors February 5, 1973 and, in revised form, May 25, 1973.

AMS (MOS) subject classifications (1970). Primary 46B05.

Key words and phrases. Vector measures, smooth Banach spaces, Grothendieck spaces, topological tensor products, Radon-Nikodym derivatives, integral operators.

Copyright $\odot$ 1974, American Mathematical Society 
of a scalar function with respect to a vector measure having values in a Banach space with Schauder basis is presented.

$\$ 4$ is concerned with the Radon-Nikodym property for Banach spaces. Contained in this section are new sufficient conditions for a Banach space to possess the Radon-Nikodym property; in particular, the classical theorem of Dunford-Pettis is generalized (Corollary 4.7).

In our concluding section, some open questions are presented.

It is with real pleasure that we thank Professor W. B. Johnson for many illuminating discussions about Banach space theory and particularly for our knowledge of the results of [1] and [46]. We are also indebted to Professor N. J. Kalton for comments concerning Theorem 1.1 and the elimination of several unnecessary hypotheses therein, and to Peter Dierolf for his comment on Corollary 1.4.

The authors thank the referee for his helpful comments and for the proof of Corollary 3.1 in its present form.

1. Strongly bounded vector measures. Let $X$ be a Banach space and $X^{*}$ denote the dual space of $X$. Let $\Omega$ be a set and $\Sigma$ be a sigma-algebra of subsets of $\Omega$.

We say that an additive map $\mu: \Sigma \rightarrow X$ is strongly bounded whenever given a sequence $\left(A_{n}\right)$ of pairwise disjoint members of $\Sigma$, one has $\mu\left(A_{n}\right) \rightarrow 0$. If $\mu$ is strongly bounded (from $\Sigma$ to $X$ ) then $\mu$ is "almost" countably additive: in fact, $\Sigma_{n} \mu\left(A_{n}\right)$ exists for any sequence $\left(A_{n}\right)$ of pairwise disjoint members of $\Sigma$. Of course, the strong boundedness of $\mu$ differs from countable additivity due to the possibility that while $\Sigma_{n} \mu\left(A_{n}\right)$ converges, it need not converge to $\mu\left(\bigcup_{n} A_{n}\right)$.

The notion of strong boundedness was introduced by C. E. Rickart in 1943 and is readily seen to provide a simultaneous generalization of the concepts of countable additivity and boundedness of variation for an additive set function. In [43], Rickart extended several classical measure-theoretic results to the class of strongly bounded set functions; most notable perhaps of Rickart's results was the demonstration of the fact that the Lebesgue decomposition theorem holds for strongly bounded set functions (see also [55]). The utility of the notion of strong boundedness has been further evidenced in recent years.

In [4], R. G. Bartle introduced a general theory of (bilinear) vectorial integration with respect to a certain class of bounded additive maps-this class is now known to coincide with the class of strongly bounded maps (see [10], [26] and [55]). Such classical results as the Vitali-Hahn-Saks theorem [10], Nikodym's theorem [10], Phillips' lemma ([10] and [26]), the Yosida-Hewitt decomposition theorem [55] and the Bartle-Dunford-Schwartz theorem (on domination by a scalar measure) ([10] and [26]) find their natural setting in the notion of strong boundedness. The theory of strongly bounded set functions has been instrumental in characterizing the weakly compact subsets in certain spaces of vector measures (see [11] for example) and has provided a source for several interesting results in the theory of Banach spaces. 
To illustrate the last assertion above we note the following: let $\lambda$ be a finite measure and $L_{\infty}(\lambda)$ denote the Banach space of essentially (with respect to $\lambda$ )bounded measurable functions (scalar-valued). Suppose $u_{n}: L_{\infty}(\lambda) \rightarrow X$ are weakly compact linear mappings $(n \geq 1)$ and suppose further that for each $f \in L_{\infty}(\lambda), \lim _{n} u_{n}(f)$ exists. Then $u_{0} f \equiv \lim _{n} u_{n}(f)$ (which by the BanachSteinhaus theorem is a bounded linear operator of $L_{\infty}(\lambda)$ to $\left.X\right)$ defines a weakly compact operator on $L_{\infty}(\lambda)$. Indeed, for each $n \geq 0$, there exists $\mu_{n}: \Sigma \rightarrow X$, bounded, additive, vanishing on $\lambda$-null sets such that

$$
u_{n} f=\int f d \mu_{n}
$$

(see [17]), for all $f \in L_{\infty}(\lambda)$ : It is known (see [26] for example; also see [15] where a different proof is presented) that the weak compactness of $u_{n}$ is equivalent to the strong boundedness of $\mu_{n}$. Thus for each $n \geq 1, \mu_{n}$ is strongly bounded; by the fact that $u_{0} f=\lim _{n} u_{n} f$ we have that for each measurable set $A, \mu_{0}(A)$ $=u_{0}\left(c_{A}\right)=\lim _{n} u_{n}\left(c_{A}\right)=\lim _{n} \mu_{n}(A)$ (where $c_{A}$ denotes the characteristic function of $A$ ). But Corollary 1.2 of [12] yields that the strong boundedness of $\mu_{n}$ implies that of $\mu_{0}$. Thus $\mu_{0}$ is strongly bounded yielding $u_{0}$ weakly compact. This result motivated the results of [15] where it is shown that this phenomenon characterizes the so-called Grothendieck spaces among all Banach spaces.

The question naturally arises as to the existence of simple criteria for bounded additive $X$-valued maps to be strongly bounded. This problem, in case the domain is an algebra, was solved in [13] and [14]: every bounded, additive, $X$ valued map defined on an algebra is strongly bounded if and only if $X$ contains no (linear homeomorphic) copy of $c_{0}$. The present section provides the corresponding result in case a sigma-algebra domain, namely, $X$ contains no copy of $l_{\infty}$. It is also noted that in this case (and only in this case) can the classical result of Pettis on weak and strong measures be improved. Particular consequences of our results are the results of Diestel [13] and Thomas [52]. Corollary to the main result is a characterization of when $X^{*}$-valued maps which are weak-star countably additive are norm countably additive. Finally, we establish criteria for a selfadjoint operator on a Hilbert space to be compact in terms of the strong boundedness (or countable additivity) of its indefinite spectral integral.

Theorem 1.1. The following statements concerning $X$ are equivalent:

(i) $X \nsupseteq l_{\infty}$, i.e., no subspace of $X$ is linearly homeomorphic to $l_{\infty}$;

(ii) given any sigma-algebra $\Sigma$ of subsets of a set $\Omega$ and any bounded additive map $\mu$ : $\Sigma \rightarrow X$, then $\mu$ is strongly bounded;

(iii) given any sigma-algebra $\Sigma$ of subsets of a set $\Omega$ and any additive map $\mu: \Sigma \rightarrow X$ and a total family $H \subseteq X^{*}$, if $x^{*} \mu$ is countably additive for each $x^{*} \in H$ then $\mu$ is countably additive.

Proof. (i) implies (ii). Denote by $B(\Omega, \Sigma)$ the linear space of bounded, real valued, $\Sigma$-measurable functions defined on $\Omega$. Norm $B(\Omega, \Sigma)$ via sup norm and 
order $B(\Omega, \Sigma)$ in the usual pointwise manner. Then $B(\Omega, \Sigma)$ is an abstract $M$ space with unit in the sense of Kakutani (see [30]). Thus $B(\Omega, \Sigma)$ is isometrically isomorphic (as a Banach lattice) with some space $C(K)$ of continuous, real-valued functions defined on a (unique up to homeomorphism) compact, Hausdorff space $K$. Now $B(\Omega, \Sigma)$ is easily seen to be Dedekind $\sigma$-complete (every countable family bounded above has a least upper bound) so by the isomorphism of $B(\Omega, \Sigma)$ with $C(K), C(K)$ is also Dedekind $\sigma$-complete. By the results of [51, Theorem 15], $K$ must be $\sigma$-Stonian, i.e., the closures of open $F_{\sigma}$-subsets of $K$ are open.

Two consequences of the above discussion are noteworthy. First, by a result of [2, Theorem 1 (Remark), p. 401], $B(\Omega, \Sigma)$ is a Grothendieck space, i.e., weak-star and weak sequential convergence in $B(\Omega, \Sigma)^{*}$ coincide; second, by a result of Rosenthal (Theorem 2.3 of [46]) every operator from $B(\Omega, \Sigma)$ to $X$ (assumed to contain no copy of $l_{\infty}$ ) is weakly compact. Thus to complete the proof of (i) implies (ii), it suffices to note the equivalence of the strong boundedness of $\mu: \Sigma \rightarrow X$ to the weak compactness of $\int d \mu: B(\Omega, \Sigma) \rightarrow X$ which is proved in [13, Theorem 2.2].

(ii) implies (iii). Let $H$ be a total subfamily of $X^{*}$ and suppose $\mu: \Sigma \rightarrow X$ is an additive set function such that $x^{*} \mu: \Sigma \rightarrow$ reals is countably additive for each $x^{*} \in H$. Then, by a theorem of Grothendieck (see [52, Proposition 0.5] for a proof), $\mu$ is bounded. Thus, by (ii), $\mu$ is strongly bounded. It follows that if $\left(A_{n}\right)$ is a sequence of pairwise disjoint members of $\Sigma$, then. $\Sigma_{n} \mu\left(A_{n}\right)$ converges in $X$. Moreover, for each $x^{*} \in H$ we have $x^{*}\left(\mu\left(\cup_{n} A_{n}\right)-\Sigma_{n} \mu\left(A_{n}\right)\right)=0$ so that by totality of $H$ in $X^{*}$ we have $\mu\left(\cup_{n} A_{n}\right)=\Sigma_{n} \mu\left(A_{n}\right)$, i.e., $\mu$ is countably additive.

Finally, (iii) implies (i). Suppose $X$ contains $l_{\infty}$. Then by a result of [40, Corollary 7.3], there is a closed linear subspace $Y$ of $X$ such that $X=l_{\infty} \oplus Y$. Note that if $H_{0}$ is any total family in $l_{\infty}^{*}$, then $H_{0} \oplus Y^{*}$ is a total family in $X^{*}=l_{\infty}^{*} \oplus Y^{*}$ (recall the pairing of $l_{\infty} \oplus Y$ and $l_{\infty}^{*} \oplus Y^{*}$ is given by $((x, y)$, $\left.\left(x^{*}, y^{*}\right)\right)=x^{*} x+y^{*} y$; Köthe, see [31]). Thus to show that (iii) fails, it suffices to exhibit a sigma-algebra $\Sigma$, an additive noncountably additive map $\mu: \Sigma \rightarrow l_{\infty}$ and a total family $H_{0} \subseteq l_{\infty}^{*}$ such that $x^{*} \mu$ is countably additive for each $x^{*} \in H_{0}$.

Such an example is produced as follows: let $\Sigma$ be the collection of all subsets of the natural numbers $N$. Define $\mu: \Sigma \rightarrow l_{\infty}$ by $\mu(A)=c_{A}$. Clearly $\mu$ is bounded, additive and nonstrongly bounded. In particular, $\mu$ is not countably additive. However, choosing $H_{0}=l_{1}$ (identified with its canonical image in $l_{\infty}^{*}=l_{1}^{* *}$ ) one obtains a total family $H_{0}$ in $l_{\infty}^{*}$ such that $x^{*} \mu$ is countably additive (verification is easy) for each $x^{*} \in H_{0}$.

Remark. The above result contains the main results of [13, Theorem 1.8] where the hypotheses that $X$ be separable or contain no copy of $c_{0}$ were shown to yield (ii). It also contains certain results of [52, Theorems $0.2,0.4]$ where the hypotheses that $X$ be separable or contain no copy of $c_{0}$ were shown to yield (iii).

Of course, the above result also sharpens (in the case that $X \nsupseteq l_{\infty}$ ) the classical theorem of B. J. Pettis asserting that weak measures are norm measures. 
Corollary 1.2. The following statements are equivalent:

(i)* $X^{*} \nsupseteq l_{\infty}$;

(ii)* every weak-star countably additive set function defined on a sigma-algebra with values in $X^{*}$ is norm countably additive.

Proof. (i)* implies (ii)*. If $\mu: \Sigma \rightarrow X^{*}$ is weak-star countably additive on the sigma-algebra $\Sigma$, then the Banach-Steinhaus theorem yields $\mu$ to be bounded; $\mu$ is clearly additive. Thus by (i)* and (i) implies (iii) of the theorem $(H=X)$ we have (ii)*.

(ii)* implies (i)*. We show that if $X^{*}$ contains a subspace isomorphic to $l_{\infty}$, then there is a weak-star countably additive nonstrongly bounded (hence noncountably additive) map on a sigma-algebra with values in $X^{*}$. Now $X^{*}$ contains a copy of $l_{\infty}$, but perhaps the first copy of $l_{\infty}$ we find in $X^{*}$ is not the best one, i.e., maybe the $X$-topology of $X^{*}$ relativized to $l_{\infty}$ is not the same as the $l_{1}$-topology of $l_{\infty}$. Then we can find another copy of $l_{\infty}$ in $X^{*}$ where the weak-star topology does relativize properly. Indeed, by Theorem 4 of [6], $X^{*}$ containing $l_{\infty}$ yields that $X$ contains a complemented copy of $l_{1}$. Thus $X$ is representable in the form $X=l_{1} \oplus Y$ for some closed linear subspace $Y$ of $X$. It follows that $X^{*}$ $=l_{1}^{*} \oplus Y^{*}=l_{\infty} \oplus Y^{*}$ and for this copy (the complement of $Y^{*}$ ) of $l_{\infty}$ the weak-* topologies match properly. Thus we can suppose that $X^{*}$ contains $l_{\infty}$ in such a manner that weak-star topologies $\left(X^{*}\right.$ with the $X$-topology and $l_{\infty}$ with its $l_{1}$-topology) coincide. Now the example of the theorem above (in proving that (iii) implies (i)) is the desired example.

Corollary 1.3. Let $(\Omega, \Sigma, \lambda)$ be a finite positive measure space and $X$ be a Banach space whose dual $X^{*}$ contains no copy of $l_{\infty}$. Let $f: \Omega \rightarrow X^{*}$ be $\lambda$-measurable and suppose that for each $x \in X, f(\cdot) x \in L_{1}(\lambda)$. Then $f$ is $\lambda$-Pettis integrable.

Proof. Recall

1. For $f$ to be $\lambda$-Pettis integrable means that given $E \in \Sigma$ there exists $f_{E} \in X^{*}$ such that for each $F \in X^{* *}$

$$
F f_{E}=\int_{E} F(f(\omega)) d \lambda(\omega)
$$

holds; see [39] for a discussion of the Pettis integral.

2. An easy application of the closed graph theorem yields that if $f(\cdot) x \in L_{1}(\lambda)$ for each $x \in X$ then, for each $E \in \Sigma$, there exists $f_{E} \in X^{*}$ such that

$$
f_{E} x=\int_{E} f(\omega)(x) d \lambda(\omega)
$$

holds for each $E \in \Sigma$. The map $\mu: \Sigma \rightarrow X^{*}$ given by $\mu(E)=f_{E}$ is weak-star countably additive. Since $X^{*}$ contains no copy of $l_{\infty}$, Corollary 1.2 guarantees that $\mu$ is norm countably additive.

Set $E_{n}=\{\omega:\|f(\omega)\| \leq n\}$. Then $f$ is Bochner integrable on every measurable subset of each $E_{n}$ and, by the countable additivity of $\mu$, 


$$
\mu(E)=\lim _{n \rightarrow \infty} \int_{E \cap E_{n}} f d \lambda \text { in norm for } E \in \Sigma .
$$

Consequently, for $E \in \Sigma$ and $F \in X^{* *}$

$$
F \mu(E)=\lim _{n \rightarrow \infty} \int_{E \cap E_{n}} F f d \lambda .
$$

Now if $|F f| \notin L_{1}(\lambda)$, there would be a set $A$ on which $F f$ is of constant sign (say positive for definiteness) such that $\int_{A} F f d \lambda=+\infty$. But then

$$
F \mu(A)=\lim _{n \rightarrow \infty} \int_{A \cap E_{n}} F f d \lambda=+\infty
$$

by the monotone convergence theorem. Hence $F f \in L_{1}(\lambda)$ for all $F \in X^{* *}$ and by (1)

$$
F \mu(E)=\int_{E} F f d \lambda
$$

for all $E \in \Sigma$. Hence $f$ is Pettis integrable.

Corollary 1.4. Let $H$ be a Hilbert space and $T: H \rightarrow H$ be a selfadjoint operator with spectral resolution $T=\int \lambda d E_{\lambda}$. If $T$ is compact then the indefinite spectral integral $\int_{B} \lambda d E_{\lambda}$, as a map from the Borel sets of the real numbers into $B(H)$, is countably additive.

Proof. If $T$ is a compact selfadjoint operator, then by the compactness of $T$, one has that given $\varepsilon>0$ and $B \in \mathscr{B}$ there exists $M$ such that

$$
\left\|\int_{B} \lambda d E_{\lambda}-\sum_{i=1}^{M} \lambda_{i}\left(E_{\lambda_{1}-0}\right)\right\|<\varepsilon,
$$

where $\left(\lambda_{n}\right)$ is the sequence of eigenvalues of $T$ and sum involves only those $\lambda_{i} \in B$. Consequently, $\left\{\int_{B} \lambda d E_{\lambda}: B \in \mathscr{B}\right\}$ is a separable subset of $B(H)$. Thus $\mu$ defined as above has separable range which of course cannot contain isomorphically $l_{\infty}$ a nonseparable space. Theorem 1.1 now applies to yield $\mu$ strongly bounded. Since, by definition of the spectral integral, given $\phi, \psi \in H, \int_{A} \lambda\left(d E_{\lambda} \varphi\right.$, $\psi$ ) is countably additive, we have $\int_{A} \lambda d E_{\lambda}$ countably additive in the norm topology of $C(H)$; indeed, the collection $\varphi \otimes \bar{\psi}$ constitutes a total family in the space $N(H)$ of nuclear operators on $H$ which is, by the results of [48, p. 48], the dual of $C(H)$. One need only now apply the reasoning of (ii) implies (iii) to yield the desired countable additivity.

2. The Jordan decomposition theorem for vector measures. Let $(\Omega, \Sigma)$ be a measurable space and let $Y$ be an order complete Banach lattice. The present section considers the problem of when a given additive map $\mu: \Sigma \rightarrow Y$ is the difference of positive additive maps.

Our first result is in response to some questions of Professor W. J. Davis; it is a fairly direct consequence of some results of Grothendieck [24] but still seems not to be widely known: 
Theorem 2.1. Suppose $Y$ is an abstract L-space [29]. Then an additive map $\mu: \Sigma \rightarrow Y$ is the difference of two nonnegative additive maps if and only if $\mu$ is of bounded variation.

Proof. Suppose $\mu: \Sigma \rightarrow Y$ is an additive map which is the difference of two positive additive maps $\mu^{+}, \mu^{-}$. Then given any finite sequence $A_{1}, \ldots, A_{n}$ of pairwise disjoint members of $\Sigma$, we have

$$
\begin{aligned}
\sum_{k=1}^{n}\left\|\mu\left(A_{k}\right)\right\| & =\sum_{k=1}^{n}\left\|\mu^{+}\left(A_{k}\right)-\mu^{-}\left(A_{k}\right)\right\| \\
& \leq \sum_{k=1}^{n}\left\|\mu^{+}\left(A_{k}\right)\right\|+\sum_{k=1}^{n}\left\|\mu^{-}\left(A_{k}\right)\right\| \\
& =\left\|\sum_{k=1}^{n} \mu^{+}\left(A_{k}\right)\right\|+\left\|\sum_{k=1}^{n} \mu^{-}\left(A_{k}\right)\right\| \\
& =\left\|\mu^{+}\left(\bigcup_{k=1}^{n} A_{k}\right)\right\|+\left\|\mu^{-}\left(\bigcup_{k=1}^{n} A_{k}\right)\right\| \\
& \leq\left\|\mu^{+}(\Omega)\right\|+\left\|\mu^{-}(\Omega)\right\|
\end{aligned}
$$

so that $\mu$ is of bounded variation.

Conversely, suppose $\mu: \Sigma \rightarrow Y$ is an additive map of bounded variation. By Kakutani's representation theorem, $Y$ is isomorphic (as a Banach lattice) to an $L_{1}(\eta)$-space for some Radon measure $\eta$ on some locally compact Hausdorff space $\Lambda$. By the results of [16], $\int d \mu: B(\Omega, \Sigma) \rightarrow Y$ is an integral operator (in the terminology of [24]). By [24, p. 142], $\int d \mu$ maps the unit ball of $B(\Omega, \Sigma)$ into an order interval in $Y$. Thus, $\int d \mu$ maps every order bounded subset of $B(\Omega, \Sigma)$ into an order interval of $Y$. Thus by Schaefer [47, p. 207], $\int d \mu$ is decomposable into the difference of two positive linear operators $u^{+}, u^{-}: B(\Omega, \Sigma) \rightarrow Y$. Let $\mu^{+}(A)$ $=u^{+}\left(c_{A}\right)$ and $\mu^{-}(A)=u^{-}\left(c_{A}\right)$ and we have decomposed $\mu$ as a difference of positive additive set functions.

Theorem 2.2. Let $\Sigma$ be the sigma-algebra of all subsets of the set $\Omega$. Let $\mu: \Sigma \rightarrow c_{0}$ be countably additive. Then $\mu$ is decomposable into the difference of positive and negative countably additive maps $\mu^{+}, \mu^{-}: \Sigma \rightarrow c_{0}$.

Proof. For each $A \subseteq \Omega$, let $\mu_{n}(A)$ denote the $n$th coordinate of $\mu(A)$. Then $\mu_{n}: \Sigma \rightarrow$ reals is countably additive and $\mu_{n}(A) \rightarrow 0$ (for each $A \in \Sigma$ ) as $n \rightarrow \infty$. By Rosenthal's generalization of Phillips' lemma [46, Lemma 1.1], it follows that $\left|\mu_{n}\right|(A) \rightarrow 0$ as $n \rightarrow \infty$ where $\left|\mu_{n}\right|(A)$ denotes the variation of $\mu_{n}$ on $A$ (we have herein used the countable additivity of $\mu$ ). Thus if we let $\tilde{\mu}(A)$ be given by

$$
\tilde{\mu}(A)=\left(\left|\mu_{n}\right|(A)\right)
$$

then $\tilde{\mu}: \Sigma \rightarrow c_{0}$ is well defined and nonnegative valued. We note that $\tilde{\mu}$ is countably additive by Nikodym's theorem $\left(\tilde{\mu}(A)=\lim _{n} \sum_{k=1}^{n}\left|\mu_{n}\right|(A) e_{n}\right.$ where $e_{n}$ is the usual $n$th unit vector in $\left.c_{0}\right)$, and $\mu(A) \leq \tilde{\mu}(A)$ for each $A \in \Sigma$. Thus, 
$\mu(A)=\tilde{\mu}(A)-(\tilde{\mu}(A)-\mu(A))$ for each $A \in \Sigma$, and $\mu$ is decomposed as difference of the countably additive measures $\mu^{+}=\tilde{\mu}$ and $\mu^{-}=\tilde{\mu}-\mu$.

Remark. It is easily seen that if the additive map $\mu: \Sigma \rightarrow Y$ (any Banach lattice) is decomposable into positive and negative parts, then $\mu(\Sigma)$ is order bounded in $Y$. In particular, if the additive map $\mu: \Sigma \rightarrow c_{0}$ is decomposable into the difference of positive additive set functions then $\mu(\Sigma)$ must be relatively compact in $c_{0}$. Thus Theorem 2.2 is indeed peculiar to sigma-algebras of all subsets of a given set as the following example demonstrates.

Let $\Omega=[0,2 \pi], \Sigma$ denote the collection of Lebesgue measurable subsets of $\Omega$ and define $\mu: \Sigma \rightarrow c_{0}$ by

$$
\mu(A)=\left(\frac{1}{(2 \pi)^{1 / 2}} \int_{A} \sin N t+d t\right) ;
$$

$\mu$ is well defined by the Riemann-Lebesgue lemma and is easily seen to be countably additive and of finite variation. However, the range of $\mu$ is easily seen to be nonrelatively compact so that $\mu$ cannot be decomposable into the difference of positive and negative additive set functions. Incidentally, the nonrelative compactness of $\mu$ 's range also establishes the non-Bochner differentiability of $\mu$ with respect to Lebesgue measure.

3. Integrability with respect to a vector measure. Let $(\Omega, \Sigma)$ be a measurable space, $X$ be a Banach space and $\mu: \Sigma \rightarrow X$ be countably additive.

This section is devoted to developing criteria for integrability of a scalar-valued function defined on $\Omega$ with respect to $\mu$. We use the integration theory developed in [5].

Theorem 3.1. Let $s: \Omega \rightarrow$ scalars be $\mu$-measurable. Then there exist a $\mu$ measurable, $\mu$-essentially bounded function $\bar{s}: \Omega \rightarrow$ scalars, a sequence $\left(E_{n}\right)$ of pairwise disjoint members of $\Sigma$ and a sequence $\left(s_{n}\right)$ of scalars such that for $\mu$-almost all $\omega \in \Omega$,

$$
s(\omega)=\Sigma_{n} s_{n} c_{E_{n}}(\omega)+\bar{s}(\omega) .
$$

Moreover, $s$ is $\mu$-integrable if and only if for each $E \in \Sigma$, the series $\Sigma_{n} s_{n} \mu\left(E \cap E_{n}\right)$ is unconditionally convergent; in this case,

$$
\int_{E} s d \mu=\Sigma_{n} s_{n} \mu\left(E \cap E_{n}\right)+\int_{E} s d \mu
$$

holds for each $E \in \Sigma$.

Proof. The representability of $s$ in the form (*) can be found in Corollary 1 , p. 73 of Hille-Phillips [58].

Recall from [5] that $s: \Omega \rightarrow$ scalars is $\mu$-integrable if there exists a sequence $\bar{s}_{n}: \Omega \rightarrow$ scalars of $\Sigma$-simple functions converging $\mu$-almost everywhere to $s$ such that for each $E \in \Sigma$, the sequence $\left(\int_{E} \bar{S}_{n} d \mu\right)$ is convergent; in this case, $\int_{E} s d \mu=\lim _{n} \int_{E} \bar{s}_{n} d \mu$. 
Now suppose that $s$ is $\mu$-integrable. Then since every $\mu$-measurable $\mu$-essentially bounded function on $\Omega$ is $\mu$-integrable [5, Theorem 2.6] and since the class of $\mu$ integrable functions is linear, $s-\bar{s}=\Sigma_{n} s_{n} c_{E_{n}}$ is $\mu$-integrable, and since the integral is linear $\int_{E} s d \mu-\int_{E} \bar{s} d \mu=\int_{E} \Sigma_{n} s_{n} c_{E_{n}} d \mu$ holds for each $E \in \Sigma$. But now by the countable additivity of the indefinite integral of a $\mu$-integrable function we have

$$
\begin{aligned}
\int_{E} \Sigma_{n} s_{n} c_{E_{n}} d \mu & =\int_{E \cap\left(U_{n} E_{n}\right)} \Sigma_{m} s_{m} c_{E_{m}} d \mu \\
& =\Sigma_{n} \int_{E \cap E_{n}}^{j} \Sigma_{m} s_{m} c_{E_{m}} d \mu \\
& =\Sigma_{n} \int_{E \cap E_{n}} s_{n} c_{E_{n}} d \mu \\
& =\Sigma_{n} s_{n} \mu\left(E \cap E_{n}\right)
\end{aligned}
$$

for each $E \in \Sigma$, where implicit in the above calculation is the conclusion that $\Sigma_{n} s_{n} \mu\left(E \cap E_{n}\right)$ converges (because of the unordered nature of the sequence $\left(E_{n}\right)$ necessarily the convergence is unconditional).

Now suppose that $\Sigma_{n} s_{n} \mu\left(E \cap E_{n}\right)$ converges unconditionally for each $E \in \Sigma$. As above, we need only show that $\Sigma_{n} s_{n} c_{E_{n}}$ is $\mu$-integrable (our reasoning above shows that $\int_{E} \Sigma_{n} s_{n} c_{E_{n}} d \mu$ must be $\left.\Sigma_{n} s_{n} \mu\left(E \cap E_{n}\right)\right)$. But this is immediate: let $\bar{s}_{n}=\sum_{k=1}^{n} s_{k} c_{E_{k}}$. As $\lambda\left(\cup_{k=n+1}^{\infty} E_{k}\right) \rightarrow 0$ as $n \rightarrow \infty, s_{n}$ converges to $\Sigma_{n} s_{n} c_{E_{n}} \lambda$ almost everywhere ( $\lambda$ is as in the first paragraph), hence $\mu$-almost everywhere. Moreover, given $E \in \Sigma, \int_{E} \bar{s}_{n} d \mu=\sum_{k=1}^{n} s_{k} \mu\left(E \cap E_{k}\right)$, a convergent sequence by hypothesis. Thus, $\Sigma_{n} s_{n} c_{E_{n}}$ is $\mu$-integrable and $\int_{E} \Sigma_{n} s_{n} c_{E_{n}}=\Sigma_{n} s_{n} \mu\left(E \cap E_{n}\right)$ holds as desired.

We now are able to prove the main result of the present section. First we recall from [38] the following definition: a linear continuous operator $u: X \rightarrow Y(X, Y$ are Banach spaces) is said to be unconditionally converging whenever given a series $\Sigma_{n} x_{n}$ in $X$ such that $\Sigma_{n}\left|f x_{n}\right|<\infty$ for all $f \in X^{*}$ then the series $\Sigma_{n} u x_{n}$ is unconditionally convergent in $Y$. In [38, Corollary 4] it is shown that if $X$ is a $C(K)$-space then $u: X \rightarrow Y$ is unconditionally converging if and only if $u$ is weakly compact; if either $X$ or $Y$ is without a copy of $c_{0}$ then by the results of [6, Theorem 5] it follows that every operator $u: X \rightarrow Y$ is unconditionally converging.

Theorem 3.2. Let $u: X \rightarrow Y$ be linear, continuous. Then $u$ is unconditionally converging if and only if given any measurable space $(\Omega, \Sigma)$ and any countably additive $\mu: \Sigma \rightarrow X$ if s: $\Omega \rightarrow$ scalars is f $\mu$-integrable, $\mu$-measurable for all $f \in X^{*}$ then $s$ is up-integrable.

Proof. Suppose $u$ is unconditionally converging and let $\mu: \Sigma \rightarrow X$ be countably additive and $s: \Omega \rightarrow$ scalars be $f \mu$-integrable and $\mu$-measurable. Then, by Theorem 3.1, there exist a $\mu$-measurable, $\mu$-essentially bounded function $\bar{s}: \Omega \rightarrow$ scalars, a sequence $\left(E_{n}\right)$ of pairwise disjoint members of $\Sigma$ and a sequence of 
scalars $\left(s_{n}\right)$ such that for $\mu$-almost all $\omega \in \Omega$,

$$
s(\omega)=\Sigma_{n} s_{n} c_{E_{n}}(\omega)+\bar{s}(\omega) .
$$

Clearly this representation also holds for the measure $u \mu: \Sigma \rightarrow Y$. By Theorem 3.1 it suffices (in showing $s$ is $u \mu$-integrable) to show that for each $E \in \Sigma$,

$$
\Sigma_{n} s_{n} u \mu\left(E \cap E_{n}\right)
$$

is unconditionally convergent in $Y$. This is done as follows: $s$ is hypothesized to be $|f \mu|$-integrable for each $f \in X^{*}$; thus, by Theorem 3.1, for each $E \in \Sigma$, and each $f \in X^{*}$,

$$
\Sigma_{n} s_{n}|f \mu|\left(E \cap E_{n}\right)
$$

is an unconditionally (hence absolutely) convergent series of scalars. Now, it follows that

$$
\begin{aligned}
\Sigma_{n}\left|f\left(s_{n} \mu\left(E \cap E_{n}\right)\right)\right| & =\Sigma_{n}\left|s_{n} f\left(\mu\left(E \cap E_{n}\right)\right)\right| \\
& \leq \Sigma_{n}\left|s_{n}\right||f \mu|\left(E \cap E_{n}\right)<\infty .
\end{aligned}
$$

Hence, by $u$ 's unconditionally convergent nature applied to the series $\Sigma_{n} x_{n}$ where $x_{n}=s_{n} \mu\left(E \cap E_{n}\right)$,

$$
\Sigma_{n} u\left(s_{n} \mu\left(E \cap E_{n}\right)\right)=\Sigma_{n} s_{n} u \mu\left(E \cap E_{n}\right)
$$

is unconditionally converging.

Now suppose that $u: X \rightarrow Y$ is not unconditionally converging. Then by (Pelczyński [38]) there exists a subspace $X_{0}$ of $X$ isomorphic to $c_{0}$ such that $\left.u\right|_{x_{0}}$ is an isomorphism (so that $X_{0}$ and $u X_{0}$ are both isomorphic to $c_{0}$ ). Thus to prove the converse one readily sees that it suffices to show that there exist a measurable space $(\Omega, \Sigma)$, a measure $\mu: \Sigma \rightarrow c_{0}$ and a $\mu$-measurable function $s: \Omega \rightarrow$ scalars such that $s$ is $|f \mu|$-integrable for each $f \in c_{0}^{*}\left(=l_{1}\right)$ yet $s$ is not $\mu$-integrable.

For $\Omega$, choose $(0,1)$; for $\Sigma$, choose the collection of Lebesgue measurable subsets of $(0,1)$; let $\lambda$ denote Lebesgue measure; define for $A \in \Sigma, \mu(A)$ $=\left(\lambda\left(A \cap A_{n}\right)\right)$ where $A_{n}=\left(1 / 2^{n+1}, 1 / 2^{n}\right)$ and define $s: \Omega \rightarrow$ scalars by $s(\omega)$ $=\Sigma_{n} c_{A_{n}}(\omega) \lambda\left(A_{n}\right)^{-1}$. That $s$ and $\mu$ fit the bill is quickly verified.

Corollary (D. R. Lewis [32]). A necessary and sufficient condition that for any measure $\mu$ (on a sigma-algebra) with values in $X$ a function which is scalarly $\mu$ integrable be $\mu$-integrable is that $X$ contain no copy of $c_{0}$.

The example constructed in Theorem 3.2 of a scalar function that is scalarly integrable with respect to a vector measure but not integrable is somewhat informative. Note in particular that if one considers for each $n$ the measure $\mu_{n}: \Sigma \rightarrow c_{0}$ defined by $\mu_{n}(A)=\left(\lambda(A \cap A), \ldots, \lambda\left(A \cap A_{n}\right), 0,0, \ldots\right)$ then, for each $A \in \Sigma, \mu_{n}(A) \rightarrow \mu(A)$. Also, the function $s$ is $\mu_{n}$-integrable but the sequence 
$\left(\int_{A} s d \mu_{n}\right)$ is not uniformly countably additive. That this is the heart of the matter (concerning the $\mu$-integrability of $s$ ) is demonstrated in

Theorem 3.3. Let $\mu_{n}, \mu: \Sigma \rightarrow X$ be countably additive, $\mu_{n} \ll \mu$, and suppose that for each $A \in \Sigma, \mu_{n}(A) \rightarrow \mu(A)$. Then if $s$ is $\mu_{n}$-integrable and $\mu$-measurable (s: $\Omega \rightarrow$ scalars) in order that $s$ be $\mu$-integrable with $\int s d \mu=\lim _{n} \int s d \mu_{n}$ for each $A \in \Sigma$ it is necessary and sufficient that $\left(\int s d \mu_{n}\right)$ be uniformly countably additive.

Proof. Necessity is an immediate consequence of Nikodym's theorem (see [12, Corollary 2.2]). To prove sufficiency, we start by representing $s$ in the form

$$
s(\omega)=\Sigma_{m} s_{m} c_{E_{m}}(\omega)+\bar{s}(\omega)
$$

where $\left(s_{m}\right)$ is a scalar sequence, $\left(E_{m}\right)$ is a sequence of pairwise disjoint members of $\Sigma$ and $s: \Omega \rightarrow$ scalars is a $\mu$-measurable $\mu$-essentially bounded function and (*) holds for $\mu$-almost all $\omega \in \Omega$ also.

Consider the Banach space (essential supremum norm) $B$ of all $\mu$-measurable, $\mu$-essentially bounded functions $t: \Omega \rightarrow$ scalars. Each $\mu_{n}$ (and $\mu$ ) acts on $B$ (via integration) in a continuous linear fashion as an operator from $B$ to $X$. The norm of the induced operators is given by the semivariation of the generating measures (see [17, p. 141]). By the hypothesis that $\mu_{n}(A) \rightarrow \mu(A)$ for each $A \in \Sigma$ and by the Dieudonné-Grothendieck boundedness theorem [52, Lemma 0.6] the $\mu_{n}$ 's and $\mu$ have uniformly bounded semivariations (thus the corresponding operators are uniformly bounded). The condition $\mu_{n}(A) \rightarrow \mu(A)$ ensures that the operators induced by the $\mu_{n}$ 's converge to the operator induced by $\mu$ at each simple function. The density of this latter class in $B$, the uniform boundedness of the operators and the pointwise convergence on the dense subspace imply by [19, II.3.6] that the operators converge at each $\xi \in B$; in particular we have that for each $E \in \Sigma$

$$
\int_{E} \bar{s} d \mu=\lim _{n} \int_{E} \bar{s} d \mu_{n} .
$$

Thus by Nikodym's theorem [12, Corollary 2.2$]\left(\int_{(\cdot)} \bar{s} d \mu_{n}\right)$ is uniformly countably additive. Thus $\left(\int_{(\cdot)}(s-\bar{s}) d \mu_{n}\right)$ is uniformly countably additive.

Let $\varepsilon>0$ be given. Then if $E \in \Sigma$ is given, by the uniform countable additivity of the sequence $\left(\int_{(\cdot)} s-\bar{s} d \mu_{n}\right)$ we have the existence of $N_{\varepsilon}$ such that

$$
\sup _{\Delta \subseteq\left\{N_{\varepsilon}+1, \ldots\right\}}\left\|\int_{E \cap U_{k \in \Delta} E_{k}} s-\bar{s} d \mu_{n}\right\| \leq \varepsilon
$$

holds for all $n$. Thus, for all $n$ and all $j \geq k \geq n$

$$
\left\|\sum_{m=k}^{j} s_{m} \mu_{n}\left(E \cap E_{m}\right)\right\| \leq \varepsilon .
$$

By hypothesis $\mu_{n}(A) \rightarrow \mu(A)$ for each $A \in \Sigma$ so 


$$
\left\|\sum_{m=k}^{j} s_{m} \mu\left(E \cap E_{m}\right)\right\| \leq \varepsilon
$$

holds as well for $j \geq k \geq N_{e}$, i.e., $\Sigma_{n} s_{n} \mu\left(E \cap E_{n}\right)$ is unconditionally convergent. The integrability of $s$ with respect to $\mu$ now follows from Theorem 3.1. A similarly simple argument shows that $\lim _{n} \Sigma_{m} s_{m} \mu_{n}\left(E \cap E_{m}\right)=\Sigma_{m} s_{m} \mu\left(E \cap E_{m}\right)$ from which one concludes that $\int_{E} s d \mu=\lim _{n} \int_{E} s d \mu_{n}$ holds for each $E \in \Sigma$.

Corollary. Let $X$ be a Banach space which possesses a Schauder basis $\left(x_{n}, f_{n}\right)$ and let $\mu: \Sigma \rightarrow X$ be countably additive. Let $\pi_{n}: \Sigma \rightarrow X$ be defined by $\pi_{n}(A)$ $=\sum_{k=1}^{n} f_{k}(\mu(A)) x_{k}$. Let s: $\Omega \rightarrow$ scalars be $\mu$-measurable, f $\mu$-integrable for each $f \in X^{*}$. Then $s$ is $\mu$-integrable and $\int_{E} s d \mu=\Sigma_{n}\left(\int_{E} s d f_{n} \mu\right) x_{n}$ holds if and only if $\left(\int_{(\cdot)} s d \pi_{n}\right)$ is uniformly countably additive.

4. On the Radon-Nikodym property for Banach spaces. As in the scalar case, the Radon-Nikodym theorem plays a central role in the development of the theory of vector-valued measures. Contrary, however, to the scalar situation a vector measure of bounded variation need not have a (Bochner) derivative with respect to its variation. Whenever each $X$-valued vector measure of bounded variation has a (Bochner) derivative with respect to its variation we say the Banach space $X$ possesses the Radon-Nikodym property (RNP); an equivalent formulation: $X$ possesses RNP if and only if given any positive finite measure space $(\Omega, \Sigma, \lambda)$ and any $\lambda$-continuous countably additive map $\mu: \Sigma \rightarrow X$ of finite variation there exists a $\lambda$-Bochner integrable $x: \Omega \rightarrow X$ such that $\mu(A)=\int_{A} x(\omega) d \lambda(\omega)$ holds for $A \in \Sigma$.

The classical results pertaining to the RNP are those of N. Dunford and B. J. Pettis (every separable dual Banach space possesses RNP) and N. Dunford, B. J. Pettis and R. S. Phillips (every reflexive Banach space possesses RNP).

The results went largely unused until the Memoir of A. Grothendieck [24] applied these results to conclude (among other things) that if a Banach space possesses the approximation property and is either reflexive or a separable dual space then it possesses the metric approximation property. The proof of this implication was dependent upon the above results of Dunford-Pettis-Phillips. That these implications do not obtain in general has only recently been shown by T. Figiel and W. B. Johnson [20].

Recent years have evidenced an increased interest in the study of vector measures and particularly the study of the Radon-Nikodym theorem. Largely through the work of M. Metivier [36], M. A. Rieffel ([44], [45]) and J. J. Uhl, Jr. ([23], [37], [54], [56], [57]) necessary and sufficient conditions for a vector measure to be differentiable with respect to a scalar measure have been obtained and new applications of the theory of vector measures have been indicated. Perhaps the most intriguing of the Radon-Nikodym theorems proved is the "dentable theorem" due to M. A. Rieffel, introducing as it does the geometry of a Banach space into the question of whether or not a given measure has a derivative. This 
result of Rieffel lead $\mathrm{H}$. Maynard [35] to the first internal characterization of the RNP for a Banach space.

Theorem 4.1 (Maynard). A Banach space $X$ possesses RNP if and only if every bounded subset of $X$ is $\omega$-dentable.

Using an observation of Maynard's to the effect that a set $D$ is $\omega$-dentable whenever every countable subset of $D$ is $\omega$-dentable one can readily prove the following

Theorem 4.2 (Uhl [56]). Let $X$ be a Banach space.

(1) The following are equivalent:

(i) $X$ possesses $R N P$;

(ii) every closed subspace of $X$ possesses $R N P$;

(iii) every separable closed subspace of $X$ possesses $R N P$.

(2) If every separable closed subspace of $X$ possesses a separable dual space then $X^{*}$ possesses $R N P$.

We shall use Theorem 4.2 (2) to derive most of our results.

Our first (and main) result of the present section is geometric in nature. Recall that a Banach space $X$ is said to be smooth whenever each $x \in X$ of norm one has a unique support functional $f_{x} \in X^{*}$; if $X$ is smooth then the map $x \rightarrow f_{x}$ of $\{x \in X:\|x\|=1\}$ into $\left\{f \in X^{*}:\|f\|=1\right\}$ is norm to weak-star continuous (see [21] for an excellent exposition of the basic facts dealing with smoothness). We say that $X$ is very smooth whenever this support mapping $x \rightarrow f_{x}$ is norm to weak continuous.

Theorem 4.3. Let $X$ be a Banach space with an equivalent very smooth norm. Then $X^{*}$ possesses the Radon-Nikodym property.

Proof. In light of the remarks preceding the theorem and the isomorphic invariance of RNP, it suffices to show that every separable subspace of $X$ (normed by its equivalent very smooth norm) has a separable dual space.

Let $S$ be a separable subspace of the very smooth space $X$. Then $S$ is very smooth: this is an immediate consequence of the Hahn-Banach theorem and the fact that smoothness of $X$ implies that support functionals in $S^{*}$ of members of $S$ must extend in a unique fashion to support functionals in $X^{*}$. To show that $S^{*}$ is separable it suffices to show that the unit sphere in $S^{*}$, i.e., $\left\{f \in S^{*}:\|f\|=1\right\}$, contains a weakly dense sequence (this is an easy consequence of Mazur's theorem [19, V. 2.14]). Let $D$ be the subset of $\left\{f \in S^{*}:\|f\|=1\right\}$ consisting of those $f$ 's that achieve their norm on the unit ball $\{x \in S:\|x\| \leq 1\}$ of $S$. Recall that by the Bishop-Phelps theorem [8], $D$ is norm dense in the unit sphere of $S^{*}$. Thus we need only show that $D$ is weakly separable. Let $C$ be a countable dense subset of $\{x \in S:\|x\|=1\}$. Let $f \in D$. Then there exists $x_{f} \in S$ such that $f x_{f}=1$, where $\left\|x_{f}\right\|=1$. Let $\left(x_{n}\right)$ be a sequence of members of $C$ such that $\left\|x_{f}-x_{n}\right\| \rightarrow 0$. Then letting $\phi x_{n}$ be the unique support functionals of $x_{n}$ we have 
by the very smooth nature of \|\| that $\phi x_{n} \rightarrow \phi x_{f}$ (weakly) $=f$. Thus $\phi C$ is a countable weakly dense subset of $D$. Proof is complete.

As every Banach space $X$ with an equivalent Fréchet differentiable norm (i.e., smooth space with the support map $x \rightarrow f_{x}$ norm to norm continuous) is very smooth, Theorem 4.3 yields the following result of E. Leonard and K. Sundaresan [60]:

Corollary 4.4. If $X$ is a Banach space with an equivalent Fréchet differentiable norm then $X^{*}$ possesses the Radon-Nikodym property.

It should be noted that in [60] more information than just Corollary 4.4 is obtained, namely, the explicit form of the support maps images is determined for the classical Lebesgue-Bochner function spaces.

It is naturally of interest to determine what (if any) notion of rotundity is dual to that of a very smooth Banach space. In view of the concern of this section with the Radon-Nikodym property the next result holds particular interest.

A Banach space $X$ is said to be weakly locally uniformly convex [33] whenever given $x_{n}, x_{0} \in X,\left\|x_{n}\right\|=1=\left\|x_{0}\right\|$ and $\left\|x_{n}+x_{0}\right\| \rightarrow 2$ then $x_{n} \rightarrow x_{0}$ (weakly).

Theorem 4.5. Suppose $X^{*}$ is weakly locally uniformly convex (in the dual norm inherited from the Banach space $X)$. Then $X$ is very smooth.

Consequently, weakly locally uniformly convex duals possess the Radon-Nikodym property.

Proof. We first note that every weakly locally uniformly convex space $Y$ is strictly convex. Indeed, if $y_{1}, y_{2} \in Y$ and $\left\|\lambda y_{1}+(1-\lambda) y_{2}\right\|=1$ for $0 \leq \lambda \leq 1$ then letting

$$
z_{n}=(1 / n) y_{1}+(1-1 / n) y_{2}
$$

we have $\left\|z_{n}\right\|=1$ and $z_{n} \rightarrow y_{2}$. Moreover,

$$
\left\|y_{1}+z_{n}\right\|=2\left\|\frac{(1+1 / n)}{2} y_{1}+\frac{(1-1 / n)}{2} y_{2}\right\|=2 .
$$

Thus $z_{n} \rightarrow y_{1}$ (weakly) from which we can conclude that $y_{1}=y_{2}$ and the strict convexity of $Y$ is proved.

It follows now from a result of Klee [59] that because $X^{*}$ is strictly convex, $X$ is smooth. Let $x \rightarrow f_{x}$ denote the support mapping given by $X$ 's smoothness. Suppose $x_{0}, x_{n} \in X,\left\|x_{0}\right\|=1=\left\|x_{n}\right\|$ and $\left\|x_{0}-x_{n}\right\| \rightarrow 0$. Then $f_{x_{n}} \rightarrow f_{x_{0}}$ (weak-star); in particular, $f_{x_{n}}\left(x_{0}\right) \rightarrow f_{x_{0}}\left(x_{0}\right)=1$. But then

$$
2 \geq\left\|f_{x_{n}}+f_{x_{0}}\right\| \geq\left(f_{x_{n}}+f_{x_{0}}\right)\left(x_{0}\right) \rightarrow 2
$$

so that $\left\|f_{x_{n}}+f_{x_{0}}\right\| \rightarrow 2$. By the weak local uniform convexity of $X^{*}$, it follows that $f_{x_{n}} \rightarrow f_{x_{0}}$ (weakly). Thus $X$ is very smooth as advertised. 
A Banach space $X$ is said to be locally uniformly convex [34] whenever given $x_{n}, x_{0} \in X,\left\|x_{n}\right\|=1=\left\|x_{0}\right\|$ and $\left\|x_{n}+x_{0}\right\| \rightarrow 2$ then $\left\|x_{0}-x_{n}\right\| \rightarrow 0$. It is immediate from Theorem 4.5 that we have

Corollary 4.6. Locally uniformly convex duals possess the Radon-Nikodym property.

Corollary 4.7. Let $\Omega$ be an infinite compact Hausdorff F-space (see [50]). Then $C(\Omega)$ cannot be renormed smoothly.

In particular, if $\Omega$ is an extremally disconnected compact Hausdorff space then $C(\Omega)$ cannot be renormed smoothly.

Proof. G. L. Seever [50, Theorem 2.5] has shown that if $\Omega$ is a compact Hausdorff $F$-space then $C(\Omega)$ is a Grothendieck space, i.e., in $C(\Omega)^{*}$ weak-star and weak sequential convergence coincide. Clearly any (equivalent) renorming of a Grothendieck space is again a Grothendieck space so that a smooth renorming of a Grothendieck $C(\Omega)$ space yields a very smooth space. By Theorem 4.3, $C(\Omega)^{*}$ possesses the Radon-Nikodym property and is, therefore, isomorphic to $l_{1}(\Gamma)$ for some set $\Gamma\left(C(\Omega)^{*}\right.$ is an $L_{1}(\mu)$-space and, as is well known, the only $L_{1}(\mu)$-spaces with the Radon-Nikodym property are isomorphic to $l_{1}(\Gamma)$-spaces). Thus $\Omega$ is dispersed. But $\Omega$ is an infinite compact Hausdorff $F$-space, hence contains $\beta N[22$, p. 215] and is, therefore, not dispersed.

Remark. With but a little more work, the above proof yields the following: let $\Omega$ be an infinite compact, Hausdorff space such that $C(\Omega)$ is a Grothendieck space; then $C(\Omega)$ cannot be renormed smoothly. The extra bit of work involves noting that every dispersed compact Hausdorff space $\Omega$ is sequentially compact [3, Proposition 1] hence contains nontrivial convergent sequences (in case $\Omega$ is infinite) while $\Omega$ 's for which $C(\Omega)$ is a Grothendieck space never contain nontrivial convergent sequences.

5. Some open questions and concluding remarks. The results of the preceding sections are far from conclusive. Somewhat indicative of the problems still open are the following:

1. Under what conditions on a quasi-complete locally convex linear topological space $E$ need bounded additive $E$-valued maps be strongly bounded?

We mention that if $E$ is separable or if $E$ contains no copy of $c_{0}$ then utilizing the results of [24] and [53] one can obtain the result that bounded and additive $E$-valued maps on sigma-algebra domains are strongly bounded. We venture to conjecture that the conclusion of Theorem 1.1 generalizes to quasi-complete locally convex spaces. In view of the application of vector measures in this direction, such a result would be interesting.

2. Given a Banach space $X$ with the Radon-Nikodym property, need every separable subspace of $X$ be isomorphic to a subspace of a separable dual?

Such has been conjectured by J. J. Uhl [56]. As is clear from the proof of Theorem 4.3, this is the crucial element present in all our results. 
It is perhaps best to point out that our geometric approach does not obviously yield the following observation of Professor Charles Stegall which improves our Corollary 4.7: if $X^{*}$ is weakly compactly generated then $X^{*}$ possesses the RadonNikodym property. Stegall's proof shows this by use of Theorem 4.2, the HahnBanach theorem and Corollary 1, p. 241 of [33]. Of course it is a well-known open question as to whether or not $X^{*}$ 's being weakly compactly generated implies $X$ is weakly compactly generated.

Question 2 and the work of Bessaga and Pełczyński [7] as well as all the known examples tend to indicate a deep connection between the bounded KreinMilman property (every closed bounded convex set has extreme points) and the Radon-Nikodym property. Thus

3. Is the bounded Krein-Milman property equivalent to the Radon-Nikodym property?

Added in proof.

Lindenstrauss has shown (summer, 1973) that the Radon-Nikodym property implies the Krein-Milman property.

R. Huff and P. Morris have shown (January, 1974) that if $X$ is a dual space and $X$ has the Krein-Milman property, then $X$ has the Radon-Nikodym property.

The most promising direction for a negative answer to Question 3 seems to be in that of tensor products. Utilizing Theorem 4.2 and Lemma 4 of [1], one can show that if $X$ and $Y$ are reflexive Banach spaces one of which possesses the approximation property, then $X \otimes_{\gamma} Y$ ( $\gamma$ denotes the greatest crossnorm of [49]) possesses the Radon-Nikodym property though $X \otimes_{\gamma} Y$ might be quite nonreflexive. To see this, first note $X \otimes_{\gamma} Y$ is a dual space since

$$
\begin{array}{rlrl}
X \otimes_{Y} Y & =N\left(X^{*}, Y\right) & & \text { (nuclear operators) [24, p. 165] } \\
& =I\left(X^{*}, Y\right) & & \text { (integral operators) [24, p. 134] } \\
& =\left(X^{*} \otimes_{\lambda} Y^{*}\right)^{*} &
\end{array}
$$

( $\lambda$ denotes the least crossnorm of [49]).

Then, if $S$ is a separable subspace of $X^{*} \otimes_{\lambda} Y^{*}$, there are separable subspaces $A \subset X^{*}, B \subset Y^{*}$ such that $S \subset A \otimes_{\lambda} B \subset X^{*} \otimes_{\lambda} Y^{*}$. By [1, Lemma 4] there are separable subspaces $A_{0} \subset X^{*}, B_{0} \subset Y^{*}$, such that $A \subset A_{0}, B \subset B_{0}$ and $A_{0}\left(B_{0}\right)$ is a complemented subspace of $X_{0}\left(Y_{0}\right)$. Thus one of $A_{0}, B_{0}$ has the approximation property and both are reflexive so using the same reasoning as in $(1),\left(A_{0} \otimes_{\lambda} B_{0}\right)^{*}$ $=A_{0}^{*} \otimes_{\gamma} B_{0}^{*}$ which is separable. Thus $S^{*}$ is separable.

Also, if $X, Y, X^{*}, Y^{*}$ are weakly compactly generated (with proper approximation assumptions), then $X^{*} \otimes_{\gamma} Y^{*}$ possesses the Radon-Nikodym property. Similarly, one can show that $l_{1}(\Gamma) \otimes_{\gamma} X$ possesses the Radon-Nikodym property whenever $X$ is reflexive on a separable dual. However, the following appears to be open: 
4. If $X, Y$ possess the Radon-Nikodym property, need $X \otimes_{\gamma} Y$ possess the Radon-Nikodym property?

Added in proof. Problem 4 has an affirmative answer if $X$ and $Y$ are dual spaces one of which has the approximation property.

Finally, in connection with Corollary 4.4 , we ask

5. If $X$ is a Grothendieck space with an equivalent (very) smooth norm, need $X$ be reflexive?

\section{REFERENCES}

1. D. Amir and J. Lindenstrauss, The structure of weakly compact sets in Banach spaces, Ann. of Math. (2) 88 (1968), 35-46. MR 37 \#4562.

2. T. Andô, Convergent sequences of finitely additive measures, Pacific J. Math. 11 (1961), 395-404. MR 25 \# 1255.

3. John W. Baker, Ordinal subspaces of compact spaces, General Topology and Appl. 3 (1973), 85-91.

4. R. G. Bartle, A general bilinear vector integral, Studia Math. 15 (1956), 337-352. MR 18, 289.

5. R. G. Bartle, N. Dunford and J. T. Schwartz, Weak compactness and vector measures, Canad. J. Math. 7 (1955), 289-305. MR 16, 1123.

6. C. Bessaga and A. Pełczyński, On bases and unconditional convergence of series in Banach spaces, Studia Math. 17 (1958), 151-164. MR 22 \#5872.

7._- On extreme points in separable conjugate spaces, Israel J. Math. 4 (1966), 262-264. MR 35 \#2126.

8. E. Bishop and R. R. Phelps, The support functionals of a convex set, Proc. Sympos. Pure Math., vol. 7, Amer. Math. Soc., Providence, R. I., 1963, pp. 27-35. MR 27 \#4051.

9. J. K. Brooks, Representations of weak and strong integrals in Banach spaces, Proc. Nat. Acad. Sci. U.S.A. 63 (1969), 266-270. MR 43 \#459.

10. - On the existence of a control measure, Bull. Amer. Math. Soc. 77 (1971), 999-1001. MR 44 \#4178.

11. Weak compactness in the space of vector measures, Bull. Amer. Math. Soc. 78 (1972), 284-287.

12. J. K. Brooks and R. S. Jewett, On finitely additive vector measures, Proc. Nat. Acad. Sci. U.S.A. 67 (1970), 1294-1298. MR 42 \#4697.

13. J. Diestel, Applications of weak compactness and bases to vector measures and vectorial integration, Rev. Roumaine Math. Pures Appl. 18 (1973), 211-224.

14.—, On the essential uniqueness of an example of C. E. Rickart, Prace Mat. 17 (1973), 263-264.

15. - Grothendieck spaces and vector measures, Vector and Operator Valued Measures and Applications, Academic Press, New York, 1973, pp. 97-108.

16. The Radon-Nikodym property and the coincidence of integral and nuclear operators, Rev. Roumaine Math. Pures Appl. 17 (1972), 1611-1620.

17. N. Dinculeanu, Vector measures, Internat. Ser. of Monographs in Pure and Appl. Math., vol. 95, Pergamon Press, New York; VEB Deutscher Verlag der Wissenschaften, Berlin, 1967. MR 34 \#601lb.

18. N. Dunford and B. J. Pettis, Linear operations on summable functions, Trans. Amer. Math. Soc. 47 (1940), 323-392. MR 1, 338.

19. N. Dunford and J. T. Schwartz, Linear operators. I: General theory, Pure and Appl. Math., vol. 7, Interscience, New York, 1958. MR 22 \# 8302.

20. T. Figiel and W. B. Johnson, The approximation property does not imply the bounded approximation property, Proc. Amer. Math. Soc. 41 (1973), 197-200. 
21. J. R. Giles, On a characterisation of differentiability of the norm of a normed linear space, J. Austral. Math. Soc. 12 (1971), 106-114. MR 44 \#2621.

22. L. Gillman and M. Jerison, Rings of continuous functions, University Series in Higher Math., Van Nostrand, Princeton, N. J., 1960. MR 22 \#6994.

23. N. E. Gretsky and J. J. Uhl, Jr., Bounded linear operators on Banach function spaces of vectorvalued functions, Trans. Amer. Math. Soc. 167 (1972), 263-277. MR 45 \#4178.

24. A. Grothendieck, Produits tensoriels topologiques et espaces nucléaires, Mem. Amer. Math. Soc. No. 16 (1955). MR 17, 763.

25.—, Sur les applications linéaires faiblement compactes d'espaces du type $C(K)$, Canad. J. Math. 5 (1953), 129-173. MR 15, 438.

26. C. Hoffman-Jorgenson, Vector measures, Math. Scand. 28 (1971), 5-32.

27. R. Huff, The Yosida-Hewitt decomposition theorem as an ergodic theorem, Vector and Operator Valued Measures and Applications, Academic Press, New York, 1973, pp. 133-139.

28. K. John and V. Zizler, A renorming of dual spaces, Israel J. Math. 12 (1972).

29. S. Kakutani, Concrete representation of abstract $(L)$-spaces and the mean ergodic theorem, Ann. of Math (2) 42 (1941), 523-537. MR 2, 318.

30.—, Concrete representation of abstract $(M)$-spaces (A characterization of the space of continuous functions), Ann. of Math. (2) 42 (1941), 994-1024. MR 3, 7; 8, 205.

31. G. Köthe, Topologische lineare Raüme. I, Die Grundlehren der math. Wissenschaften, Band 107, Springer-Verlag, Berlin, 1960; English transl., Die Grundlehren der math. Wissenschaften, Band 159, Springer-Verlag, New York, 1969. MR 24 \# A411; 40 \# 1750.

32. D. R. Lewis, On integrability and summability in vector spaces, Illinois J. Math. 16 (1972), 294-307.

33. J. Lindenstrauss, Weakly compact sets-their topological properties and the Banach spaces they generate, Symposium on Infinite Dimensional Topology, Ann. of Math. Studies, Princeton Univ. Press, Princeton, N. J., 1972.

34. A. R. Lovaglia, Locally uniformly convex Banach spaces, Trans. Amer. Math. Soc. 78 (1955), 225-238. MR 16, 596.

35. H. Maynard, A geometrical characterization of Banach spaces possessing the Radon-Nikodym property, Trans. Amer. Math. Soc. 185 (1973), 493-500.

36. M. Metivier, Martingales à valeuts vectorielles. Application à la dérivations des mesures vectorielles, Ann. Inst. Fourier (Grenoble) 17 (1967), fasc. 2, 175-208. MR 40 \#926.

37. S. Moedomo and J. J. Uhl, Jr., Radon-Nikodým theorems for the Bochner and Pettis integrals, Pacific J. Math. 38 (1971), 531-536.

38. A. Pekczyński, Banach space on which every unconditionally converging operator is weakly compact, Bull. Acad. Polon. Sci. Sér. Sci. Math. Astronom. Phys. 10 (1962), 641-648. MR 26 \#6785.

39. B. J. Pettis, On integration in vector spaces, Trans. Amer. Math. Soc. 44 (1938), 277-304.

40. R. S. Phillips, On linear transformations, Trans. Amer. Math. Soc. 48 (1940), 516-541. MR 2, 318.

41.— On weakly compact subsets of a Banach space, Amer. J. Math. 64 (1943), 108-136. MR 4, 218.

42. M. M. Rao, Smoothness of Orlicz spaces. I, II, Nederl. Akad. Wetensch. Proc. Ser. A $68=$ Indag. Math. 27 (1965), 671-690. MR 32 \#116.

43. C. E. Rickart, Decomposition of additive set functions, Duke Math. J. 10 (1943), 653-665. MR 5, 232.

44. M. A. Rieffel, Dentable subsets of Banach spaces, with applications to a Radon-Nikodym theorem, Functional Analysis (Proc. Conf., Irvine, Calif., 1966), Academic Press, London; Thompson Wash., D.C., 1967, pp. 71-77. MR 36 \#5668.

45.—, The Radon-Nikodym theorem for the Bochner integral, Trans. Amer. Math. Soc. 131 (1968), 466-487. MR 36 \#5297. 
46. H. P. Rosenthal, On relatively disjoint families of measures, with some applications to Banach space theory, Studia Math. 37 (1970), 13-36. MR 42 \#5015.

47. H. H. Schaefer, Topological vector spaces, Macmillan, New York, 1966. MR 33 \# 1689.

48. R. Schatten, Norm ideals of completely continuous operators, Ergebnisse der Math. und ihrer Grenzgebiete, Heft 27, Springer-Verlag, Berlin, 1960. MR 22 \#9878.

49. - A theory of cross spaces, Ann. of Math. Studies, no. 26, Princeton Univ. Press, Princeton, N. J., 1950. MR 12, 186.

50. G. L. Seever, Measures on F spaces, Trans. Amer. Math. Soc. 133 (1968), 267-280. MR 37 \#1976.

51. M. H. Stone, Boundedness properties in function lattices, Canad. J. Math. 1 (1949), 176-186. MR $10,546$.

52. G. E. F. Thomas, The Lebesgue-Nikodym theorem for vector valued Radon measures, Mem. Amer. Math. Soc. No. 139 (1974), 101 pp.

53. Ju. B. Tumarkin, On locally convex spaces with basis, Dokl. Akad. Nauk SSSR 195 (1970), 1278-1281 = Soviet Math. Dokl. 11 (1970), 1672-1675. MR 42 \#6575.

54. J. J. Uhl, Jr., Applications of Radon-Nikodým theorems to martingale convergence, Trans. Amer. Math. Soc. 145 (1969), 271-285. MR 40 \#4983.

55. Extensions and decompositions of vector measures, J. London Math. Soc. (2) 3 (1971), 672-676. MR 44 \#4181.

56. - A note on the Radon-Nikodym property for Banach spaces, Rev. Roumaine Math. Pures Appl. 17 (1972), 113-115.

57. - The Radon-Nikodym theorem and the mean convergence of Banach space valued martingales, Proc. Amer. Math. Soc. 21 (1969), 139-144. MR 38 \#6640.

58. E. Hille and R. S. Phillips, Functional analysis and semigroups, rev. ed., Amer. Math. Soc. Colloq. Publ., vol. 31, Amer. Math. Soc., Providence, R. I., 1957. MR 19, 664.

59. V. Klee, Convex bodies and periodic homeomorphisms in Hilbert space, Trans. Amer. Math. Soc. 74 (1953), 10-43. MR 14, 989.

60. E. Leonard and K. Sundaresan, Duality and smoothness in Lebesgue-Bochner function spaces, Trans. Amer. Math. Soc. (to appear).

Department of Mathematics, Kent State University, Kent, Oho 44242 